\title{
A Reified Interface for a Tutor on Program Debugging
}

\author{
Amruth N Kumar \\ Ramapo College of New Jersey, Mahwah, NJ 07430, USA \\ amruth@ramapo.edu
}

\begin{abstract}
In this paper, we will present two user interfaces we developed for a tutor on debugging programs. The second interface is reified with respect to the first, and is hence, better at capturing student misconceptions and promoting the development of an accurate mental model in the learner. We will discuss the rationale behind the interface, its significance, and its impact on the maintenance of the student model. We will also describe the design and implementation of the reified interface.
\end{abstract}

\section{Introduction}

We have been developing an intelligent tutoring system to help students learn $\mathrm{C}++$ programming by solving problems on specific constructs in the language. For each problem, the tutor presents a program involving the construct, and provides answering options corresponding to possible errors in the program. The learner may select one or more errors in the program before soliciting feedback. The tutor provides demand feedback, and explains the errors that may be present in the code.

The tutor maintains a cognitive model of the student that includes scores for each type of error - how many problems the student solved correctly, incorrectly, and partially for problems involving that type of error. This overlay model is used to sequence problems in the tutor.

To date, our tutoring system covers variables, scope, pointers [6], dynamic allocation [5], for loops, nested selection statements, function calls, and classes in $\mathrm{C}++$ [3]. Empirical evaluation of the tutor indicates that it helps improve student learning [4].

Figure 1 shows a snapshot of the first interface provided by the tutor. Learners study the code presented in the left panel, and identify the errors in the code by checking the boxes in the top-right panel. Learners may select more than one error for a given program. Students have found it easy to use this interface. We have tested this interface over several semesters, multiple tutors and multiple sections of courses.
We recently revised the user interface. In the next section, we will discuss the new interface, and how it is reified [1] with respect to the old one. We will discuss the rationale behind the interface, and its significance. In Section 3, we will describe how the new interface affects the maintenance of the student model. We will describe our implementation of the interface, along with an example in Section 4. We will discuss future work in Section 5 .

\section{The Reified Interface}

In the reified interface, the learner identifies the errors in the code as follows:

- The learner clicks on the line of code where (s)he believes a bug exists;

- From a menu of program objects (e.g., variables, functions), the learner selects the object to which the bug applies;

- From a submenu of possible reasons, the learner selects the reason why (s)he thinks the bug applies to the selected object.

- After identifying all the errors in the code, the learner clicks on the "Submit" button to commit his/her answers.

E.g., the learner may click on line 15 of the code, and specify that a dangling pointer error applies to the variable valuePointer, because it is being referenced before it is initialized.

The interface permits the user to unselect an error, and select more than one error in a program. For each line, the interface lists only the program objects involved in that line of code. Therefore, the interface is contextsensitive, and may be said to scaffold learning by presenting only the relevant errors. Please see Figure 2 for a snapshot of the tutor with the new interface.

This interface is reified with respect to the earlier interface because it not only expects the user to identify the error, but also the line of code and the program object to which that error applies:

- A program object may enter an error state only after a certain operation, e.g., a pointer pointing to another variable may become a dangling pointer only after the variable to which it points goes out of scope. 
Therefore, asking the user to identify the line where the error occurs helps distinguish between users who understand how the error is generated and those who do not, but guess the answer any way.

- An error may occur in more than one object, e.g,, semantic error may occur in any variable that is referenced before it is initialized. Asking the user to identify the program object that is subjected to an error once again distinguishes users who understand the error from those who try to guess the program behavior.

- A program object may enter an error state for various reasons, e.g., a pointer may become a dangling pointer because it was never initialized, because the variable it points to went out of scope, or because the heap object it points to was deallocated. Asking the user to identify the specific reason why a pointer is a dangling pointer helps confirm that the user understands the topic.

The reification of the interface makes it less likely that a user can guess the correct answer. We may quantify the effect of reification as follows: if a program contains $m$ lines of code, the probability that an uninformed user guesses the correct line is $1 / \mathrm{m}$. If $n$ objects are involved in the identified line, the probability of the user correctly guessing the correct object is $1 / n$. If $\mathrm{p}$ possible errors could occur in the identified object, the probability that the user guesses the correct error is $1 / \mathrm{p}$. Therefore, the combined probability that the user guesses the correct answer is $1 / \mathrm{mnp}$. This compares very favorably with the old interface (Figure 1), wherein, if there are $\mathrm{q}$ answering options, there is a 1/q probability that the user can guess the correct answer. Therefore, the new interface is mnp / q times more reified than the old interface. The term mnp / $\mathrm{q}$ applies to a novice, uninformed learner. As the learner progresses, the factor reduces to $\mathrm{np} / \mathrm{q}$ (when the learner begins to understand the program, and narrows down the error to a particular line of code) and finally, to $\mathrm{p} / \mathrm{q}$ (when the user learns enough to narrow down the error to a particular object).

We address the following special occasions with separate buttons:

- When the code works correctly - instead of clicking on a line of code, the learner clicks on a button provided specifically for this purpose.

- When the learner does not know the answer - a separate button is provided for the learner to indicate this. When the learner clicks on this button, (s)he is stepped through the feedback. The learner is not permitted to go to the next problem until (s)he has stepped through the entire feedback.

\subsection{The Rationale}

The objective of our tutors is to help the learner identify errors in a program that arise from using an incorrect program model. Therefore, the primary focuses of our tutors are semantic and run-time errors. The tutors consider syntax errors only insofar as they arise from using an incorrect program model. The tutors specifically do not address syntax errors such as missing semi-colons or improperly matched braces in a program, that are related only to the syntax of the program and not to the model behind it.

Our design of the reified interface is based on the following observations about programs and program models that give rise to errors:

1. Given the class of errors that our tutors address, every error in a program can be attributed to an error in one or more objects in the program.

The behavior of a program is completely determined by the behaviors and interactions of the objects in the program, such as variables, scope objects, etc. Therefore, any semantic error, run-time error or syntax error arising from using an incorrect program model can be attributed to one or more objects in the program.

2. Every error in an object can be attributed to the application of an invalid operation, given its current state.

Since an error is generated only after the operation is applied to an otherwise error-free object, identifying the operation is just as important as identifying the error itself. Identifying an operation involves identifying the statement performing the operation, and hence, the line of code containing the statement.

Therefore, in our reified interface, the user is asked to identify not only the error, but also the object and the operation that gave rise to the error. The user is prompted to identify the operation first, the object next and the specific error last. Since the learner is expected to identify the object and the operation before identifying the specific error, the interface emphasizes the use of a mental model of the program, as opposed to a listing of the program. By the same token, when used repeatedly to solve problems, the interface helps the learner develop a clearer mental model of programming.

This interface can be used in any tutor for programming and program debugging. It is independent of the programming language and is especially suited for imperative and object-oriented languages. Although we currently use it for $\mathrm{C}++$ tutors, we plan to reuse it for Java and C\# tutors in the future.

\section{The Student Model}


The tutor updates the cognitive model of the learner after each problem. If the learner's answer is $\mathrm{L}$, and the correct answer is $\mathrm{C}$, the tutor grades as follows:

- $\quad$ Correct answer $=\mathrm{L} \cap \mathrm{C}$.

- Incorrect answer $=\mathrm{L}-\mathrm{C}$.

- Missed answer $=\mathrm{C}-\mathrm{L}$.

The correct answer is used in support of the student model. The incorrect and missed answers are used to highlight weaknesses in the student model - this information helps the tutor sequence the problems.

One of the advantages of using the reified interface is better updating of the cognitive student model based on the student's performance. For instance, the learner may identify the right error, but either for the wrong program object or on the wrong line of code. If so, the learner has not only missed the correct answer, but also identified an incorrect error. Since the earlier version of the tutor asked only for the error, it would have identified this example as a correct answer. With the earlier interface, the sum of correct and incorrect scores reported for the student added up to the number of problems attempted by the student. With the new interface, it adds up to $|\mathrm{L}|+|\mathrm{C}|-|\mathrm{L} \cap \mathrm{C}|(|\mathrm{L}|$ refers to the cardinality of the set $\mathrm{L}$ ), which is greater than the number of problems attempted by the learner. This provides for a better approximation of the progress of the student.

\section{Implementation}

We use model-based reasoning to model the domain in our tutor [2]. The advantages of using model-based reasoning for domain modeling are:

- The domain model doubles as the runnable expert module;

- The resulting tutor is not restricted to a pre-defined problem set - it can tutor with problems entered by a generator or the learner.

In order to promote modularity, reuse and scalability of representation, we have been using a representation based on component-ontology [7] instead of stateontology. In component-ontological representation, the structure and behavior of a device are represented in terms of its components rather than their states.

We implemented the context-sensitive error menu as follows:

1. Each line of code in the problem model maintains a list of program objects involved in that line. When the learner clicks on a line of code, a menu containing these objects is dynamically generated for that line.

2. The model of each program object maintains a list of the possible errors that could occur in that object.
When the learner selects an object in the menu generated in Step 1, a submenu of these errors is dynamically generated for that object.

3. The learner's choices are maintained in a data structure until the learner commits the answer by clicking on the "Submit" Button (not shown in Figure 2). Every time the user clicks on a line of code, this data structure is consulted to see if some error should be displayed as having been previously selected. This enables the user to unselect an error, if necessary.

Our design is modular and scalable - when new program objects are added to the domain model, they are automatically co-opted into the user interface when applicable.

\subsection{An Example}

Consider the problem in Figure 2. The statement on line 15 is:

cout $<<$ *valuePointer;

1. The objects involved on this line are the pointer variable valuePointer, and the variable to which it points, count. When the user clicks on line 15 , a drop-down menu of these two objects is dynamically generated.

2. Currently, four possible errors are associated with pointer variables: a syntax error and three instances of dangling pointers. When the user selects valuePointer, a sub-menu of these four errors is presented to the user. The user may select any of the errors in this sub-menu.

3. The user may return to choose additional errors for the same object, on the same line, or for a different object on a different line of code. If the user returns to the same line and same object, any previously chosen errors are displayed as selected, and can be unselected.

\section{Future Work}

Clearly, the new interface is more natural than the old one - students point to the errors in the code by clicking on the code. The menu of errors presented to the learner is context-sensitive.

We plan to extend our interface to ask the learner to specify the output of the program. Once again, the learner would click on each output statement in the code, and specify the output generated by that statement. Loops must be treated as a special case for this extension.

\section{Acknowledgements}


Partial support for this work was provided by the National Science Foundation's Course, Curriculum and Laboratory Improvement Program under grant DUE0088864 .

\section{References}

[1] Corbett, A.T. and Anderson, J.R.: Knowledge decomposition and subgoal reification in the ACT programming tutor.

Proceedings of Artificial Intelligence in Education (1995), 469476.

[2] Davis, R.: Diagnostic Reasoning Based on Structure and Behavior. Artificial Intelligence, Vol. 24 (1984) 347-410.

[3] Kostadinov, R., Kumar, A.N.: A Tutor for Learning Encapsulation in C++ Classes, Proceedings of ED-MEDIA 2003 World Conference on Educational Multimedia, Hypermedia and Telecommunications, Honolulu, HI, 6/23-28/2003.

[4] Kumar, A.N.: ModelBased Reasoning for Domain Modeling in a Web-Based Intelligent Tutoring System to Help Students
Learn to Debug C++ Programs. Proceedings of Intelligent Tutoring Systems (ITS 2002), LNCS 2363, Biarritz, France, June 5-8, 2002, 792-801.

[5] Kumar, A.N.: A Tutor for Using Dynamic Memory in C++, Proceedings of 2002 Frontiers in Education Conference (FIE 2002), Boston, MA, 11/6-9/2002.

[6] Kumar, A.N.: Learning the Interaction between Pointers and Scope in C++, Proceedings of The Sixth Annual Conference on Innovation and Technology in Computer Science Education (ITiCSE 2001), Canterbury, UK, 6/25-27/2001, pp. 45-48

[7] Kumar, A.N. and Upadhyaya, S.J.: Component Ontological Representation of Function for Reasoning About Devices, Artificial Intelligence in Engineering, Vol 12 (4), October 1998, pp 399-415.

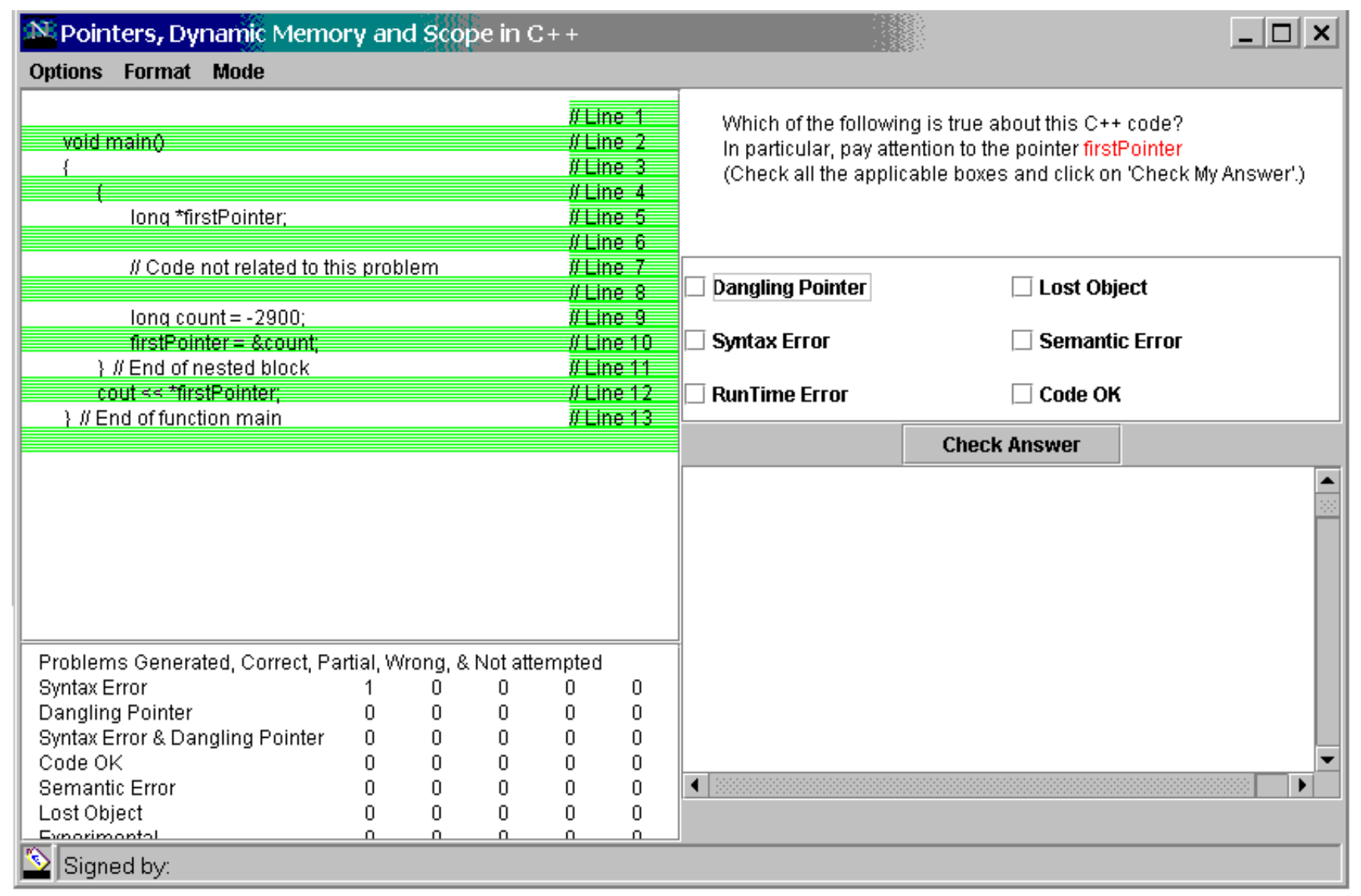

Figure 1: Snapshot of the old interface - Answering options in the right panel 


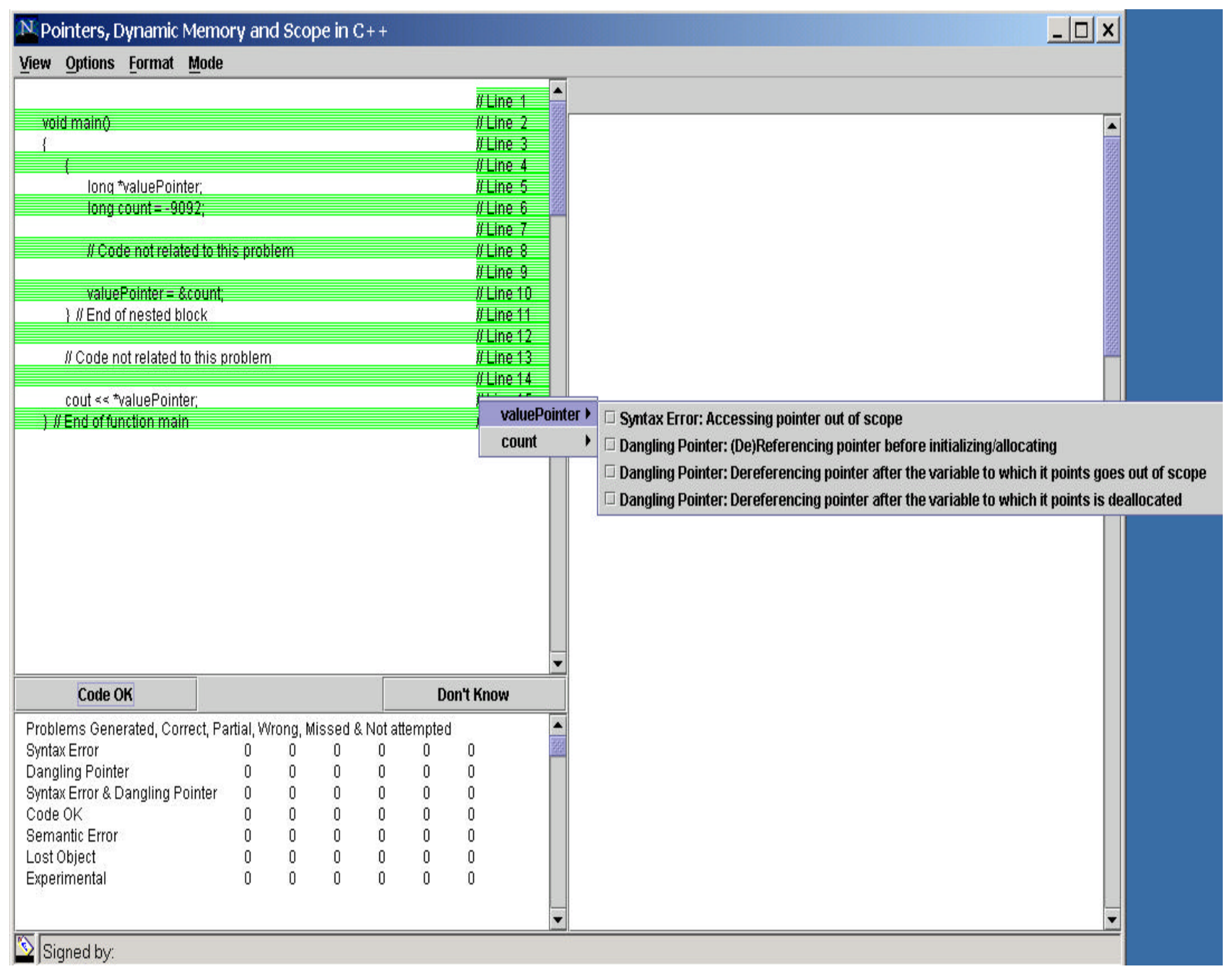

Figure 2: Snapshot of the new interface - Errors for valuePointer on line 15 are displayed 